\title{
SIMPLE MODEL OF HYSTERESIS IN THERMOELASTIC MARTENSITIC TRANSFORMATIONS
}

\author{
E. CESARI, J. PONS and C. SEGUI
}

Dept. de Fisica, Universitat Illes Balears, Ctra. de Valldemossa, km. 7.5, E-07071 Palma de Mallorca, Spain

\begin{abstract}
To attain a better understanding of the hysteretic behaviour in shape memory alloys we present in this work a simple phenomenological model based on the thermoelastic balance that must be fulfilled at every step of transformation. The model is built on the simplest choice for the chemical and non-chemical free energies, that is, a first order dependence of these functions on $T$ and $x$ (transformed fraction) respectively. From this choice, some considerations are made on the contributions to the non-chemical energy that should be taken and their physical meaning. Apart from the global hysteresis loop, the model is able to describe the subloop behaviour which, in spite of its interest from the point of view of many applications, is not well known. The study of subloops in the frame of the model leads to discuss the location of the starting temperatures for the forward and reverse partial transformations in relation to the dissipative terms.
\end{abstract}

\section{Introduction}

The hysteresis of the thermoelastic martensitic transformation has been studied both from the experimental and theoretical points of view, most of the studies concerning complete transformation-retransformation cycles $[1,2]$, although in the recent past several approaches have been made to the subloop behaviour $[3,4]$. In spite of its importance from the point of view of many applications, the real mechanisms giving rise to the hysteretic behaviour are not yet well understood, neither useful tools exist to determine "a priori" the forward/reverse transformation paths that will be followed when performing a given subloop inside the area of the global hysteresis loop.

It has been established that the main characteristics of the hysteresis loop (temperature-stress location, slopes and width) are intimately related to the energy terms contributing to the thermoelastic balance [5]. In this paper the thermoelastic balance for thermal induced stress free transformations, which must be fulfilled at every step of transformation has been used to develop a phenomenological model. which can describe complete or incomplete 
transformation cycles (under given simplifying assumptions). The model has predictive character, i.e. a subloop can be determined "a priori" once the parameters of the model have been obtained, either experimentally or theoretically.

\section{Formulation of the model}

A martensitic transformation is said to be thermoelastic in those cases in which the interfacial motion can be considered reversible at a certain macroscopic level [6]. For stress-free temperature induced transformations it means that martensite forms and grows continuously as temperature is lowered, and shrinks and vanishes continuously as temperature is increased (although most of the concepts here used are common 10 both stress-free and stress-induced transformations, only the former will be considered hereafter). A thermoelastic transformation occurs under an equilibrium condition: it is accepted that at the transforming front the total Gibbs free energy difference between martensite and parent phase is zero, $\Delta \mathrm{G}^{\mathrm{p} \rightarrow \mathrm{m}}(\mathrm{x}, \mathrm{T})=0 ; \Delta \mathrm{G}^{\mathrm{m} \rightarrow \mathrm{p}}(\mathrm{x}, \mathrm{T})=0$ for the forward and reverse transformation, respectively, where $x$ holds for the fraction of transformed volume. This energy consisting of chemical and non chemical contributions, the equilibrium condition is in fact a balance equation.

The chemical contribution becomes more negative on cooling, so it counterbalances the increasing non chemical contribution as the transformation proceeds. The non chemical contribution, in its turn, contains two types of terms, namely elastically stored strain energy and dissipative energies. The former is an essential contribution to the thermoelastic behaviour, since, being reversible in character (recoverable), it is released during the reverse transformation, on heating, acting in the same sense as the chemical driving force and allowing, in this way, the reverse transformation to occur at lower temperatures than if only chemical and dissipative energies were operative. The latter energy opposes both the forward and reverse transformations and give rise to the existence of hysteresis itself.

The thermoelastic equilibrium can be considered as a local equilibrium condition whose terms change from one to another transformation point $(x, T)$. Therefore, in a transformation step involving incremental growth of a volume fraction $d x$ of martensite, it should be accomplished that

$$
\mathrm{g}_{\mathrm{ch}}(\mathrm{x}, \mathrm{T})+\mathrm{g}_{\mathrm{nch}}(\mathrm{x}, \mathrm{T})=0
$$

where the terms indicate the energies per unit of transforming volume driving and opposing the growth of martensite at the given $(x, T)$ point. It has to be noted that only integration of those terms over the considered transformation range would lead to the total amount of chemical and non-chemical free energies exchanged (involved) in the transformation process.

The knowledge of the functional forms of the free energy terms of equation (1) would lead to the $x(T)$ path and therefore to the hysteresis loop. However, this information is not directly available neither from theoretical nor from 
experimental results. On the basis of eq. (1), the following macroscopic model attempts to approximate the hysteresis loop by means of simple functions.

The chemical contribution can be taken, as usual, in the form

$$
g_{c h}^{p \rightarrow m}(T)=-\Delta s\left(T_{0}-T\right)
$$

where $\Delta s$ stands for the absolute value of the transformation entropy change per unit volume, which will be considered to be the same for both the forward and the reverse transformations, and $T_{0}$ is the equilibrium temperature where the parent and martensite phases have the same chemical free energy in absence of any stress (that is, when $x=0$ ). The simplest approach that can be envisaged for the non chemical energies would be the use of a constant dissipative force, $\mathrm{g}_{\mathrm{d}}=\mathrm{a}^{\mathrm{p} \rightarrow \mathrm{m}}$, to be overcome at every $(\mathrm{x}, \mathrm{T})$ and, as a consequence of the increasing elastic strain energy built up in the matrix as the transformation proceeds (which gives rise to the need of continuous cooling to further move the interfaces), a term linearly dependent on $x$ for the elastic contribution, $g_{e l}=b x$ is taken. The forward transformation balance is then

$$
-\Delta s\left(T_{0}-T\right)+a^{p \rightarrow m}+b x=0
$$

It can be easily seen from (3) that $a^{p \rightarrow m} / \Delta s$ gives the undercooling below $T_{0}$ to start the forward transformation at $M s$ (for $x=0$ ) while $b / \Delta s$ gives the temperature span between $M s$ and $M f$ (between $x=0$ and $x=1$ ). The $x(T)$ path can also be obtained from (3).

The reverse transformation balance can be obtained by taking into account the reversible nature of both the chemical and elastic energies (if relaxation does not occur), so that

$$
\Delta s\left(T_{o}-T\right)+a^{m \rightarrow p}-b x=0
$$

holds at every $(x, T)$ point of the reverse transformation. An equilibrium temperature, $T_{0}$, can be defined for the reverse transformation [7], starting at $x=1$. At this point, the energy of the phases consists not only of the chemical but also the elastically stored energy, and therefore

from where it can be easily seen that

$$
\mathrm{g}_{\mathrm{ch}}+\mathrm{g}_{\mathrm{cl}}=0 \quad \text { at } \mathrm{T}=\mathrm{T}_{\mathrm{o}}
$$

$$
T_{o}^{\prime}=T_{0}-b / \Delta s
$$

From (4) the As $(x=1)$ and Af $(x=0)$ temperatures can be obtained, $a^{m \rightarrow p} / \Delta s$ giving the overheating above $T_{0}{ }^{\prime}$ to start the reverse transformation, and $b / \Delta s$ giving the Af-As difference.

The so obtained hysteresis loop is formed by straight lines with slope $b / \Delta s$ and wideness $\left(a^{p \rightarrow m}+a^{m \rightarrow p}\right) / \Delta s$. In spite of the simplicity of the linear choice, it is observed in most cases that the hysteresis loop for a thermoelastic transformation shows constant slopes on its branches over a wide range of $x$ values (between $20-80 \%$ ), and therefore the linear approach can be useful to describe this region which is thought to consist mainly of relatively free movement of the interfaces 18]. Concerning the dissipative terms, the constant parameters must be considered as an average of the dissipative energies operative during all the transformation. The nucleation phenomenon is not explicitely considered here. 
It has to be also noticed that if $\mathrm{a}^{\mathrm{p} \rightarrow \mathrm{m}}=\mathrm{a}^{\mathrm{m} \rightarrow \mathrm{p}}$, then

$$
\begin{aligned}
& T_{0}=(M s+A f) / 2 \\
& T_{0}^{\prime}=(M f+A s) / 2
\end{aligned}
$$

From the above formulation, parallel paths for the forward $(p \rightarrow m)$ and reverse $(\mathrm{m} \rightarrow \mathrm{p})$ transformations are obtained, which is not always observed in experimental hysteresis loops. The stored elastic energy being recoverable, the difference in transformation path slopes can not be accounted for by this term and has to be attributed to the existence of increasing/decreasing dissipative forces active during the martensite growth/shrinkage. To maintain the linear description of the hysteresis loop, the dissipative contributions to the thermoelastic balance can be modified so that

$$
\begin{aligned}
& g_{d}^{p \rightarrow m}=a^{p \rightarrow m}+c^{p \rightarrow m} x \\
& g_{d}^{m \rightarrow p}=a^{m \rightarrow p}+c^{m \rightarrow p}(1-x)
\end{aligned}
$$

Introducing (9) and (10) into (3) and (4), the corresponding expressions for Ms and $\mathrm{Mf}(\mathrm{x}=0$ and $\mathrm{x}=1$ respectively in the $\mathrm{p} \rightarrow \mathrm{m}$ balance) and $\mathrm{As}$, Af ( $\mathrm{x}=1$ and $\mathrm{x}=0$ respectively in the $m \rightarrow p$ balance) can be obtained. It has to be noted that Ms and As are obtained as above, while the temperature extensions Ms-Mf and AfAs depend not only on $b / \Delta s$ but also on $c^{p \rightarrow m} / \Delta s$ and $c^{m \rightarrow p} / \Delta s$ respectively. It is worth noting that, even if $a^{p \rightarrow m}=a^{m \rightarrow p}$ is taken, $T_{0}$ is no longer located halfway between $M s$ and Af, nor $T_{0}$ halfway between $A s$ and Mf.

The subloop behaviour in thermoelastic martensitic transformations can be studied from the proposed point of view, taking into account the general rules for subloop behaviour which are mainly the same for all hysteretic phenomena [9]. To give an example, let us consider an arbitrary path of direct transformation inside the global loop starting at the point $\left[\mathrm{x}_{\mathrm{s}}(\mathrm{i}), \mathrm{Ms}\left[\mathrm{x}_{\mathrm{s}}(\mathrm{i})\right]\right.$ (figure 1). To reach such a point starting from pure parent phase, several previous subloops have been performed. The parameters of the model to describe this internal path will depend on the extreme values of $x$ achieved in the previous subloops, as it occurs in the actual material behaviour. The balance equation for the considered path will be:

$$
-\Delta s(T o-T)+a^{p \rightarrow m l}(i)+b x+c^{p \rightarrow m}(i)\left\{x-x_{s}(i)\right\}=0
$$

where the dissipative energy terms, $a^{p \rightarrow m}(i)$ and $c^{p \rightarrow m}(i)$, depend on the previous extreme points achieved $\left\{x_{f}(i-1), T_{m i n}(i-1)\right\},\left\{x_{s}(i-1), \operatorname{Ms}\left(x_{s}(i-1)\right)\right\}, \ldots$

The start temperature for the forward path will correspond to $x=x_{s}(i)$ and, according to (11) is given by

$$
\operatorname{Ms}\left[x_{s}(i)\right]=T_{0}-\frac{a^{p \rightarrow m}(i)+b x_{s}(i)}{\Delta s}
$$

The location of $\left.\mathrm{Ms}_{\mathrm{s}}(\mathrm{i})\right\}$ is determined by both the dissipative forces for this particular subloop and the elastic stored energy at the starting fraction $x_{s}(i)$.

It has to be noticed that the parameter $a^{p \rightarrow m}$ (i) becomes an essential feature for determining subloop behaviour. In absence of theoretical results concerning this 
function, its choice lies on experimental results. Unfortunately, they are scarce and often contradictory. Although any a(i) form is admitted by the model, only those which do not violate the subloop rules and the physical meaning of $\mathrm{Ms}\left[\mathrm{x}_{\mathrm{s}}(\mathrm{i})\right]$ can be accepted. For the purpose of simplicity we will make a choice which consists of using a constant value $a^{p \rightarrow m}(i)=a^{p \rightarrow m}$ for all internal paths. This choice implies the existence of a unique $M s\left[x_{s}(i)\right]$ line for all subloops (the starting temperatures for all the internal paths lie on a unique straight line), as shown in figure 1 . The unicity of $\left.M s \mid x_{s}(i)\right]$ for a given $x_{s}(i)$ fraction is a'basic assumption for further development of the subloop behaviour in the frame of the model; $a^{p \rightarrow m}(i)=$ constant is not a necessary condition to achieve the $M s\left[x_{s}(i)\right]$ unicity.

Once started at $\mathrm{Ms}\left[\mathrm{x}_{\mathrm{s}}(\mathrm{i})\right]$, the internal path will tend to the previous extreme point $\left[\mathrm{x}_{\mathrm{f}}(\mathrm{i}-1), \mathrm{T}_{\min }(\mathrm{i}-1)\right]$ as is usually observed. The slope of this path is given by $\left[b+c^{p \rightarrow m}(i)\right] / \Delta s$. It is not difficult to establish that

$$
c^{p \rightarrow m}(i)=\frac{c^{p \rightarrow m} x_{f}(1)}{x_{f}(i-1)-x_{s}(i)} \prod_{j=2}^{i-1} \frac{x_{f}(j)-x_{s}(j)}{x_{l}(j-1)-x_{s}(j)}
$$

where $x_{S}(1)=0$ and $x_{f}(1)$ lie on the external (global loop) branch. Substitution into (10) allows to determine the $x(T)$ path starting from $x_{s}(i)$.

For an arbitrary $m \rightarrow p$ internal path starting from $x_{f}(i)$ (maintaining the same notation as above, the reverse transformation paths go from $x_{r}(j)$ to $x_{s}(j+1)$ ), the corresponding thermoelastic balance is

$$
\operatorname{As}\left(T_{0}-T\right)+a^{m \rightarrow p}(i)-b x+c^{m \rightarrow p}(i)\left|x_{f}(i)-x\right|=0
$$

$a^{m \rightarrow p}(i)$ will determine the location of the $A s\left|x_{i}(i)\right|$ and, according to the same considerations as before, we will use $a^{n \rightarrow p}(i)=a^{m \rightarrow p}$ constant, from where

$$
\operatorname{As}\left[x_{f}(i)\right]=T_{0}+\frac{a^{m \rightarrow p}-b x_{f}(i)}{\Delta s}
$$

which ensure the existence of a unique $A s\left[x_{[}(i)\right]$ line. Furthermore, at a given $x$ martensite fraction, an equilibrium temperature between austenite and martensite can be considered, which fulfil

$$
\begin{gathered}
g_{c h}\left(T_{0}(x)\right)+g_{0}(x)=0 \\
\text { that is: } \quad T_{0}(x)=T_{0}-\frac{b x}{\Delta s}
\end{gathered}
$$

As $\left[\mathrm{x}_{\mathrm{f}}(\mathrm{i})\right]$ as well as $\mathrm{Ms}\left\lfloor\mathrm{x}_{\mathrm{s}}(\mathrm{i})\right\rfloor$ lie always above and below $\mathrm{T}_{0}(\mathrm{x})$ respectively. It has to be noted that $T_{0}$ " as defined in (6) is a limit for (17) for $x=1$.

On heating above As[ $\left.x_{f}(i)\right]$, the reverse partial transformation path tends towards the previous extreme $\left[x_{s}(i+1), T_{\max }(i+1)\right]$. An expression similar to (13) for $c^{m \rightarrow p}(i)$ as a function of $c^{m \rightarrow p}$ and the previous extreme points $x_{s}, x_{f}$ can be obtained:

$$
c^{m \rightarrow p(i)}=\frac{c^{m \rightarrow p}}{x_{f}(i)-x_{s}(i+1)} \prod_{j=2}^{i-1} \frac{x_{l}(j)-x_{s}(j+1)}{x_{f}(j)-x_{s}(j)}
$$

which completes the set of expresions needed to determine the modelled subloop from known parameters for the global loop. Subloop behaviour, studied 
by means of calorimetry, in a $\mathrm{Cu}-\mathrm{Zn}$-Al alloy showing $\beta \leftrightarrow 2 \mathrm{H}$ transformation agree quite well with the $a^{\mathrm{m} \rightarrow \mathrm{p}}(\mathrm{i})=\mathrm{ct}$ statement.

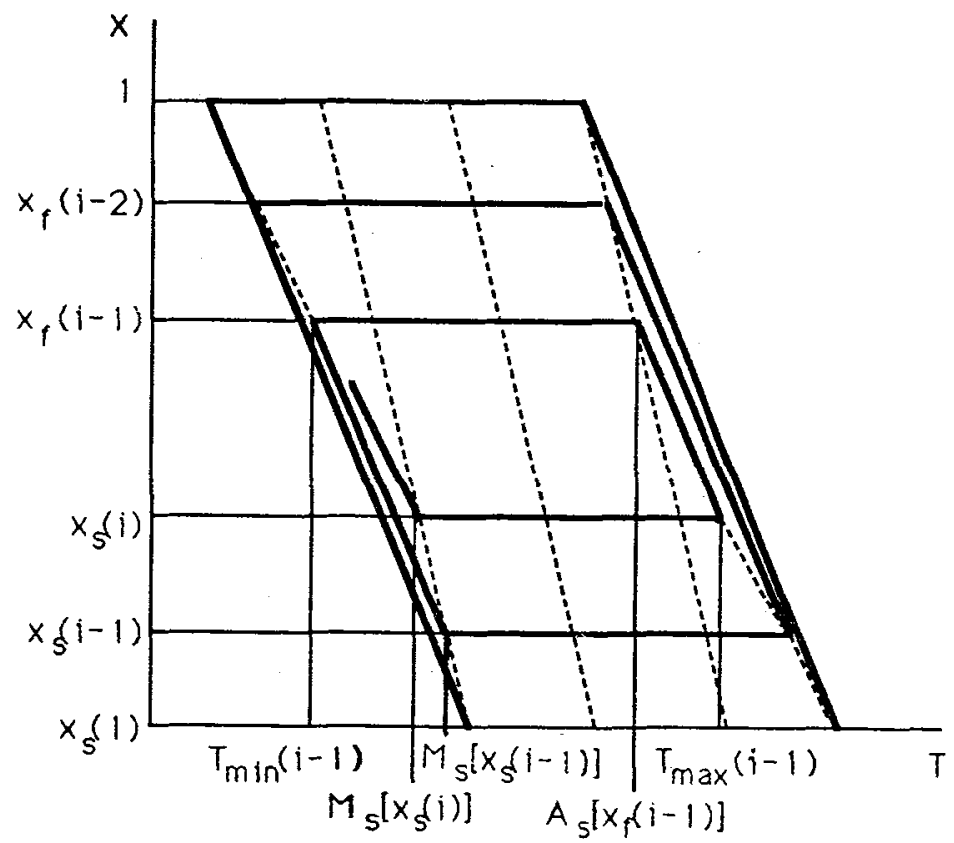

Figure 1. Schematic drawing of partial transformation paths inside the global loop. Corresponds to the case of $\mathrm{i}=3$.

\section{Acknowledgements}

J. Pons acknowledges the DGICyT of Spain for the concession of a grant of the PFPI. Partial financial support from CICyT (project num. MAT89-0407-C03-03) is also acknowledged.

\section{References}

[1] AMENGUAL A., LOVEY F.C., SEGUI C., TORRA V., Mat. Sci. Forum 56-58 (1990) 523

[2] DelaeY L., ORTIN J., VAN HUMBeECK J., Proc. of Phase Transf. 87, The Inst. of Metals, Ed. G.W. Lorimer (1988) p.60)

[3] ZHONGGUO W., DAZHL Y., Scripta Metall. 22 (1988) 1245

[4] LU L., AERNOUDT E., WOLLANTS P., VAN HUMBEECK J., DELAEY L.; $Z$. Metallkde. 81 (1990) 613

[5] SALZBRENNER R.J., COHEN M.; Acta Metall. 27 (1979) 739

[6] DELAEY L., KRISHNAN R.V., TAS H., WARLiMONT H.; J. Mater. Sci. 9 (1974) 1521

[7] TONG H.C., WAYMAN C.M., Acta Metall. 23 (1979) 209

[8] DONG Y., ANSELL G.S.; Acta Metall. 38 (1990) 69

[9] CORY J.S., McNICHOLS J.L. Jr ; J. Appl. Phys. 58 (1985) 3282 